\title{
In Vivo Targeting through Click Chemistry
}

\section{Citation}

Brudno, Yevgeny, Rajiv M. Desai, Brian J. Kwee, Neel S. Joshi, Michael Aizenberg, and David J. Mooney. 2015. "In Vivo Targeting through Click Chemistry." ChemMedChem 10 (4) (February 20): 617-620. doi:10.1002/cmdc.201402527.

\section{Published Version}

doi:10.1002/cmdc.201402527

\section{Permanent link}

http://nrs.harvard.edu/urn-3:HUL.InstRepos:14531725

\section{Terms of Use}

This article was downloaded from Harvard University's DASH repository, and is made available under the terms and conditions applicable to Open Access Policy Articles, as set forth at http:// nrs.harvard.edu/urn-3:HUL.InstRepos:dash.current.terms-of-use\#OAP

\section{Share Your Story}

The Harvard community has made this article openly available.

Please share how this access benefits you. Submit a story.

Accessibility 


\title{
In Vivo Targeting through Click Chemistry
}

\author{
Yevgeny Brudno ${ }^{[1,2]}$, Rajiv Desai ${ }^{[1,2]}$, Brian J Kwee ${ }^{[1,2]}$, Neel S Joshi ${ }^{[1,2]}$, Michael Aizenberg ${ }^{[1]}$, and David \\ J Mooney ${ }^{[1,2, *]}$
}

\begin{abstract}
Targeting small molecules to diseased tissues as therapy or diagnosis is a significant challenge in drug delivery. Drug-eluting devices implanted during invasive surgery allow for controlled presentation of drugs at disease site, but can not be modified once surgery is complete. We demonstrate that bioorthogonal click chemistry can be used to target circulating small molecules to hydrogels resident intramuscularly in diseased tissues. Additionally, we demonstrate that small molecules can repeatedly be targeted to the diseased area over the course of at least one month. Finally, two bioorthogonal reactions were used to segregate two small molecules injected as a mixture to two separate locations in a mouse disease model. These results demonstrate that click chemistry can be used for pharmacological drug delivery, and this concept is expected to have applications in refilling drug depots in cancer therapy, wound healing and drug-eluting vascular grafts and stents.
\end{abstract}

Systemic drug toxicity is a serious problem in current clinical interventions, including in cancer, arrthymia $^{[1]}$, immunosuppression $^{[2]}$ and ocular ${ }^{[3]}$ drug delivery. Local drugdelivering devices confer a substantial reduction in toxicity and thus have significant clinical utility, including in prevention of restenosis with stenting ${ }^{[4]}$, cancer treatment ${ }^{[5]}$ and enhanced wound healing ${ }^{[6]}$. In applications where repeated local implantation of devices is not possible, such as for implantation of a drug-delivering scaffold during invasive surgery, drugs would need to be targeted to implant sites through non-invasive means.

One approach to target implanted devices is to modify them with molecular targets capable of recognizing and binding small molecules circulating in the body. Accumulated small molecules bound by the implant could function in bound form, or be slowly released over time, allowing for controlled drug dosing. Here we report the utilization of bioorthogonal click chemistry to accumulate small molecules at a site of animal injury.

Recently we introduced the concept of refillable drug delivery devices in which DNA recognition was used to target drugcarrying nanoparticle refills to a device placed within a tumor site $^{[7]}$. Drug payloads circulating in the blood of a patient could be bound by the device though specific chemical recognition, allowing for subsequent release through low-pH-mediated hydrolysis ${ }^{[8]}$ or enzymatic degradation ${ }^{[9]}$ at the target site.

In this work we hypothesized that drug-eluting hydrogels could

[a] Dr. Yevgeny Brudno, Brian J. Kwee, Rajiv R. Desai, Prof. Neel S. Joshi, Dr. Michael Aizenberg, Prof. David J. Mooney

Wyss Institute for Biologically Inspired Engineering

Harvard University

3 Blackfan Circle, Boston, MA 02115

E-mail: mooneyd@seas.harvard.edu

[b] Dr. Yevgeny Brudno, Brian J. Kwee, Rajiv R. Desai, Prof. Neel S. Joshi, Prof. David J. Mooney

School of Engineering and Applied Sciences

Harvard University

29 Oxford St. Cambridge MA 02138

Supporting information for this article is given via a link at the end of the document.

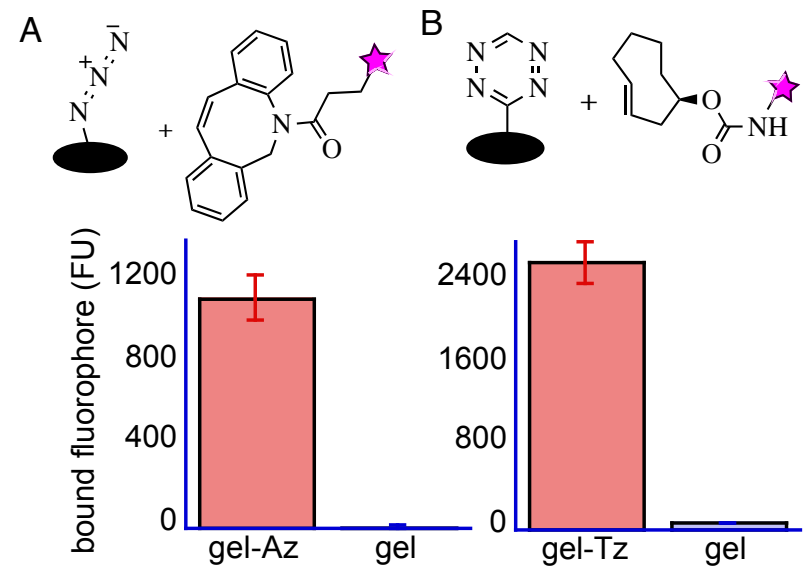

Figure 1. Click chemistry-mediated gel targeting in vitro. (A) Azide-conjugated or control, calcium-crosslinked alginate gels were incubated with fluorophore carrying $\mathrm{DBCO}$ for 4 hours at $37^{\circ} \mathrm{C}$. Retained fluorescence was quantified. (B) Tetrazine-conjugated or control, calcium-crosslinked alginate gels were incubated with fluorophore carrying trans-cyclooctene for 4 hours at $37^{\circ} \mathrm{C}$. Retained fluorescence was quantified.

be targeted by small molecules in circulation through click chemistry-mediated recognition of the hydrogels. Two click chemistries were identified as candidates for drug targeting due to high specificity, efficient kinetics and established use in vivo[10]: 1) trans-cyclooctene (TCO) - Tetrazine (Tz) chemistry and 2) dibenzocyclooctyne (DBCO) - Azide (Az) chemistry.

Alginate, a naturally occurring polysaccharide, was chosen as a drug delivery vehicle due to its clinical track record[1]. Alginate is biocompatible and non-immunogenic in intramuscular environments. Calcium carbonate-induced gelation of unoxidized, high MW alginate as used in this report creates nanoporous[2], injectable gels with a viscosity of .15-2 Pa*s[3]. These gels present minimal changes in mass upon in vivo implantation[4] and present slow degradation kinetics in vivo, still present at implant site after multiple months[3, 5]. Further addition of click groups to alginate gels has not been noted to alter biocompatibility in vivo[6].

Alginate was modified with either tetrazine or azide through cabodiimide chemistry. NMR analysis demonstrated that on average 100 molecules of Tz/Az were attached to every alginate strand, constituting 400 nanomoles of target per mg of polymer. Specific binding of fluorescently-labeled TCO and DBCO molecules to hydrogels was established in vitro. Cy7-labeled trans-cyclooctene specifically bound to tetrazine-modified alginate, but not to unmodified alginate (Figure 1A). Similarly, fluorescent DBCO bound alginate-azide gels while demonstrating little interaction with unmodified alginate gels (Figure 1B).

It was next investigated whether small molecules labeled with near-IR (NIR) fluorophores could target in vivo to gels resident at a disease site in an animal model of lower limb ischemia. $50 \mu \mathrm{L}$ of tetrazine-modified, azide-modified, or unmodified alginate gels 


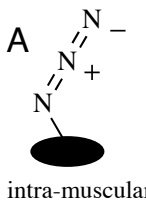

gel

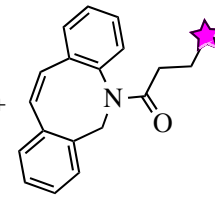

IV-injected

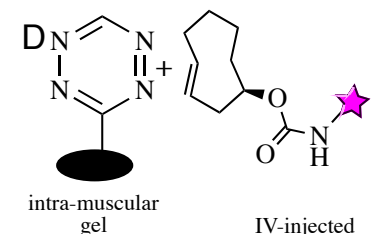

E

IV-injected
B

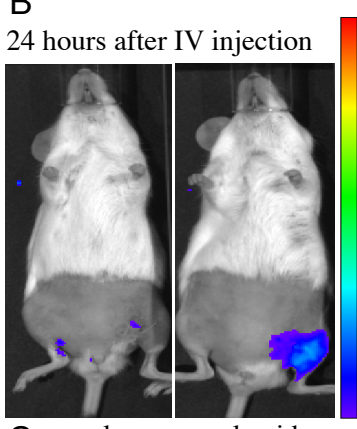

C gel
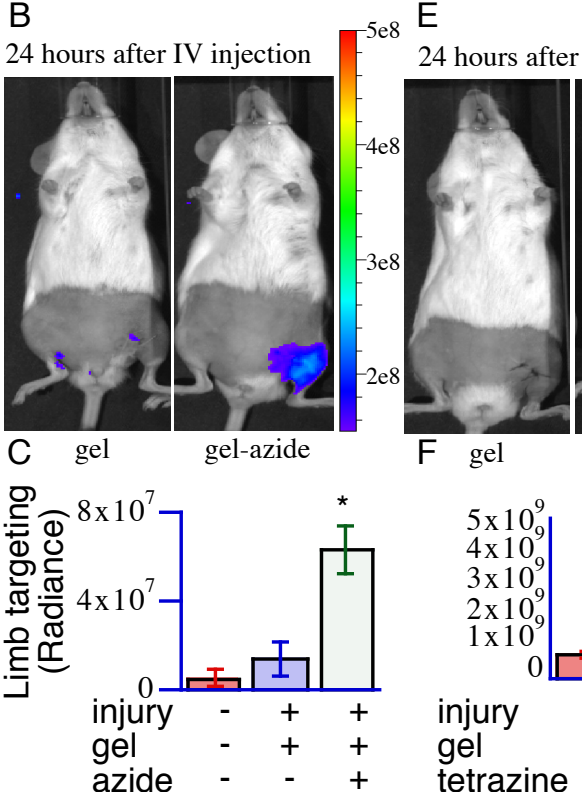

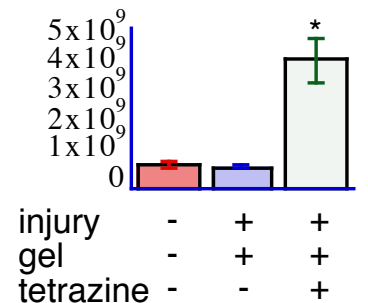

Figure 2. Click-mediated targeting of a drug surrogate to hindlimb injury model. Mice subjected to hindlimb ischemia were implanted with unconjugated alginate gels or conjugated to azide (A-C) or tetrazine (D-F). Targeting to this gel was tested through IV injection of a fluorescently-labeled DBCO (A-C) or TCO (D-F). $(A, C)$ reaction scheme $(B, E)$ images and $(C, F)$ quantification of fluorescence at the hind limb. Values represent mean and S.E.M. $n=3$. * represents $p<.05$ by Student's t-test.

were implanted intra-muscularly in the limbs of mice subjected to femoral artery ligation, in a similar manner to the use of drugdelivering alginate gels to treat animal models of peripheral

artery disease $(P A D)^{[12]}$. 24-hours post-surgery, NIR-labeled DBCO or TCO-molecules were administered intravenously (IV) and the animals were monitored over 24 hours through live animal fluorescence imaging. We found that the NIR-labeled small molecules circulated in the animal's system, and were eliminated mainly through the bladder in the first 24 hours (SI Figure 1), as expected ${ }^{[13]}$. After 24-hours, fluorescence was observed in the limbs implanted with modified gels, but not in control gels, which lacked the targeting motifs (Figure 2).

To more accurately understand the dose of circulating molecules homing to intra-muscular gels, azide-modified gels were isolated from mouse muscles and digested. The amount of targeted molecules on the gels was quantified by fluorescence. An average of 106 picomoles of targeting compound, corresponding to $6.5 \%$ of the initially injected dose, localized to the intramuscular disease area (SI Table 1). This number of molecules constitutes only $0.03 \%$ of total available sites on the gel, suggesting that multiple gel fillings would be possible.

Multiple intravascular administrations were then performed to repeatedly target and fill the intramuscular gel in injured mice. Mice with hind limb ischemia carrying azide-modified gels were repeatedly administered NIR-labeled DBCO, approximately once every three days for one month (Figure $3 \mathrm{~A}$ ). Over the one-month time course, limb fluorescence increased in a step-wise fashion, corresponding to fluorophore administrations (Figure 3B), with each dose increasing fluorescence to a similar extent (Figure 3C). A small, but not significant, decrease in fluorescence in the limb was generally observed between 24- and 48-hours after IV injection, perhaps due to residual unbound fluorophore removed from the gel.

It was hypothesized that the two molecules, DBCO and TCO, could selectively home to their respective binding partners to achieve spatial separation of drug-surrogates in the same animal. To test this, two sites on the mouse were chosen: tetrazine-modified gels were implanted intra-muscularly in the hind limb of mice subjected to hind-limb ischemia while azidemodified gels were implanted into the mammary fat pad, to simulate a second target site. A mixture of Cy7-labeled DBCO and Cy5-labeled TCO was administered intravenously 24 hours after gel administration. Efficient targeting by the two small

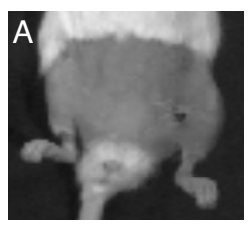

uninjected

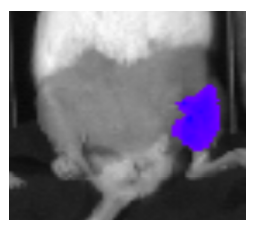

injection \#1

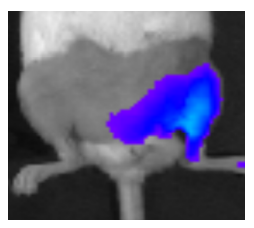

injection \#3

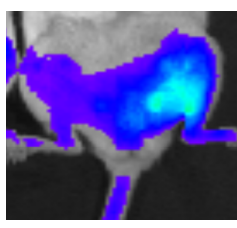

injection \#5

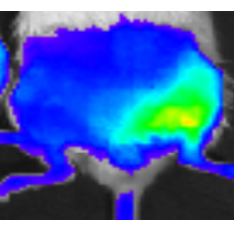

injection \#7

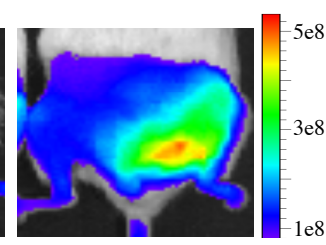

injection \#9
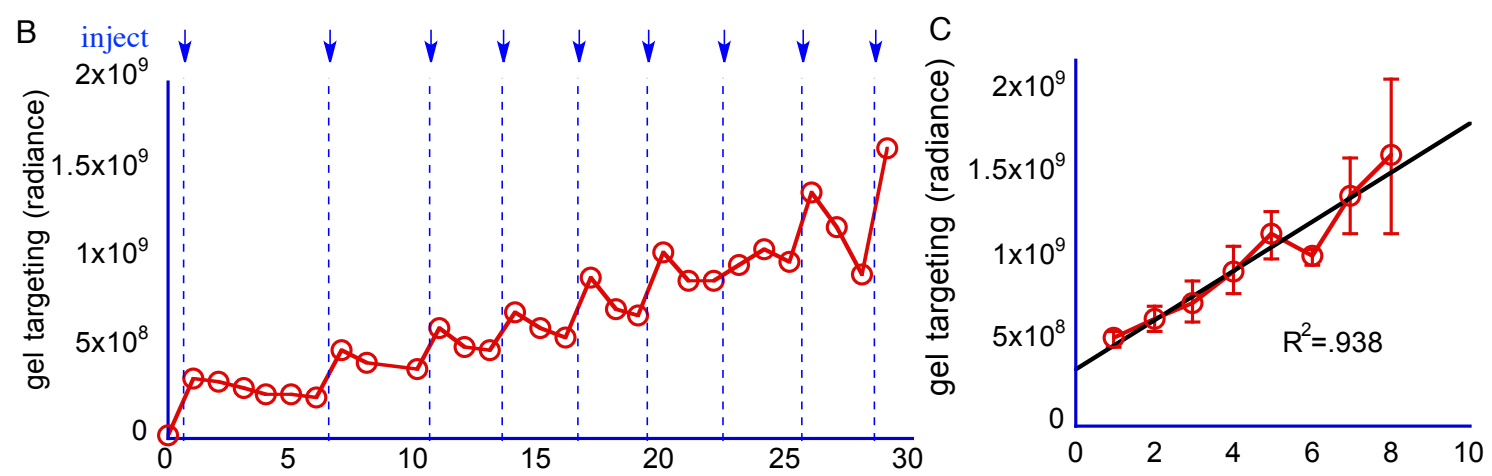

Figure 3. Repeat targeting of fluorescent drug surrogates to intra-muscular gel. Fluorescently-labeled DBCO was repeatedly administered to mice (blue arrows) over the course of a one month. (A) Images and (B) quantitation of limb fluorescence 24 hours after repeat injections of targeting fluorescent DBCO. (C) Quantitation of fluorescence 24-hours after fluorophore administration shows a roughly linear increase in fluorescence with each injection. 
molecules to their respective disease sites was observed (Figure 4). Though targeting of hydrogels was previously demonstrated $^{[10 b]}$, here we demonstrate gel homing within an animal model of ischemia. Additionally, we show repeated homing to the gel site over a period of one month and the ability to segregate two different molecules through the use of two orthogonal chemistry systems. The ability to repeatedly target drug surrogates to a specific site within the body suggests that medical implants, such as hydrogels and medical devices, can be selectively targeted by small molecules circulating in the blood.

The stability of tetrazines in serum is dependent on the precise chemical structure of tetrazine. The tetrazine used in this report is reported to retain $40 \%$ function over ten hours ${ }^{[14]}$, in agreement with the observation that intramuscular tetrazinemodified gels could be filled 24 hours after implanatation, but failed to be targeted seven days after implantation. Future work can take advantage of more stable tetrazines for improved stability of targeted gels ${ }^{[14]}$. Additionally, the TCO and tetrazine groups could be exchanged in the drug targeting system, placing the more stable TCO group onto the long-lasting gel. These exciting possibilities are the subject of future work.

Efficient and repeated targeting of drug depots could allow for long-lasting, local drug delivery at sites of intravascular stenting, intra-tumor chemotherapy delivery devices or surgical implants, allowing for lowered toxicity, improved dosing and drug availability at disease site. Efficient homing of drug molecules to sites defined by an implant could also allow for subsequent controlled or on-demand release by an external stimulus such as a magnetic field ${ }^{[15]}$ or focused light ${ }^{[16]}$.

In summary, we report a system for selective targeting of small molecule drug surrogates to hydrogels in both ischemic and non-ischemic locations, including in muscular and mammary sites. Click-mediated targeting exhibited a high degree of specificity for the target sites and the targeting could be repeated over a period of at least one month through nine administrations. Finally, we demonstrate spatial resolution of two
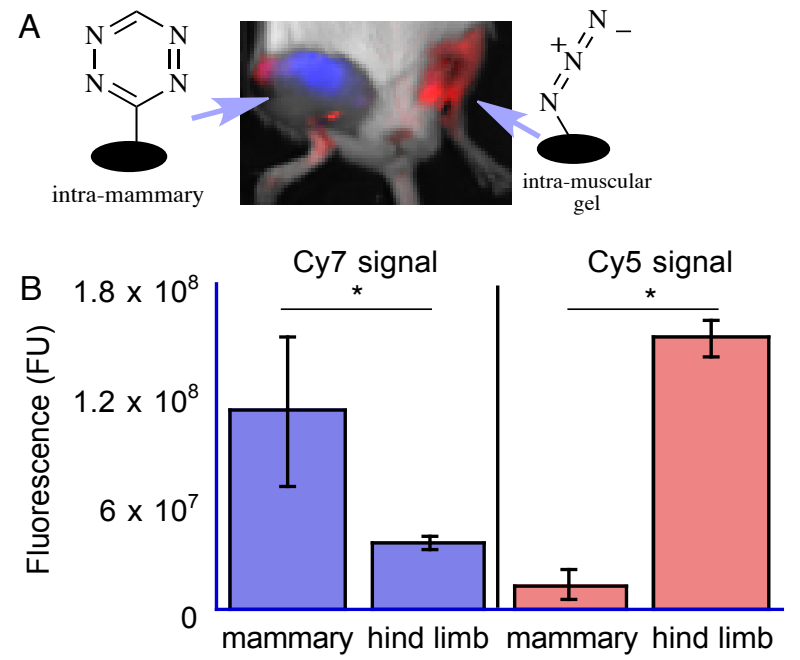

Figure 4. Spatial encoding for two different small molecules. Alginate-Az gel was implanted intra-muscularly to mice with hind limb ischemia (left limb) and alginateTz was implanted into the mammary fat pad (right body). A mixture of Cy5-TCO and Cy7-DBCO was injected IV and animals were imaged after 48 hours. (A) Cy5 (red) and Cy7 (green) fluorescence shows homing of the respective click partners. (B) Quantification of fluorescence at implantation sites for both fluorophores. Values represent mean and S.E.M. $n=3$. * represents $p<.01$ by Student's t-test. different molecules targeting two different sites in the same animal, establishing the possibility to combine incompatible therapies in patients.

\section{Experimental Section}

For detailed experimental conditions and chemical analysis see the Supporting Information

\section{Acknowledgements}

This work was supported by the Wyss Institute for Biologically Inspired Engineering. Y.B. gratefully acknowledge funding support from the Wyss Technology Development Fellowship. This work was supported by the Army Research Office (W911NF-13-1-0242) and the Wyss Institute.

Keywords: drug delivery $\bullet$ click chemistry $\cdot$ gels $\bullet$ bioorthogonal chemistry $\bullet$ drug targeting

[1] K. K. Flaharty, S. L. Chase, H. M. Yaghsezian, R. Rubin, Pharmacotherapy 1989, 9, 39-44

[2] R. R. Tamesis, A. Rodriguez, W. G. Christen, Y. A. Akova, E. Messmer C. S. Foster, Ophthalmology 1996, 103, 768-775.

[3] A. J. Flach, The Journal of the Florida Medical Association 1994, 81 , 256-260.

[4] a) T. Simard, B. Hibbert, F. Ramirez, M. Froeschl, Y.-X. Chen, E. O'Brien, The Canadian journal of cardiology 2013; b) R. Wessely, Nature reviews. Cardiology 2010, 7, 194-203.

[5] T. Iwamoto, Biological \& pharmaceutical bulletin 2013, 36, 715-718. [6] a) S. Attanasio, J. Snell, Cardiology in review 2009, 17, 115-120; b) S. Freedman, J. Isner, Journal of molecular and cellular cardiology 2001, 33, 379-393; c) D. Losordo, S. Dimmeler, Circulation 2004, 109, 2487-2491; d) Y. Brudno, A. B. Ennett-Shepard, R. R. Chen, M. Aizenberg, D. J. Mooney, Biomaterials 2013, 34, 9201-9209.

[7] Y. Brudno, E. A. Silva, C. J. Kearney, S. A. Lewin, A. Miller, K. D. Martinick, M. Aizenberg, D. J. Mooney, Proceedings of the National Academy of Sciences of the United States of America 2014

[8] a) V. Knorr, M. Ogris, E. Wagner, Pharmaceutical research 2008, 25, 2937-2945; b) N. Murthy, Y. X. Thng, S. Schuck, M. C. Xu, J. M. Frechet, Journal of the American Chemical Society 2002, 124, 12398-12399; c) M. C. Parrott, J. C. Luft, J. D. Byrne, J. H. Fain, M. E. Napier, J. M. Desimone, Journal of the American Chemical Society 2010, 132, 17928-17932; d) S. Xu, Y. Luo, R. Graeser, A. Warnecke, F. Kratz, P. Hauff, K. Licha, R. Haag, Bioorganic \& medicinal chemistry letters 2009, 19, 1030-1034.

[9] B. Turk, Nature reviews. Drug discovery 2006, 5, 785-799.

[10] a) J. M. Baskin, J. A. Prescher, S. T. Laughlin, N. J. Agard, P. V. Chang, I. A. Miller, A. Lo, J. A. Codelli, C. R. Bertozzi, Proceedings of the National Academy of Sciences of the United States of America 2007, 104, 1679316797 ; b) J. M. Mejia Oneto, M. Gupta, J. K. Leach, M. Lee, J. L. Sutcliffe, Acta biomaterialia 2014; c) A. A. Neves, H. Stockmann, Y. A. Wainman, J. C. Kuo, S. Fawcett, F. J. Leeper, K. M. Brindle, Bioconjugate chemistry 2013, 24 934-941; d) H. Koo, S. Lee, J. H. Na, S. H. Kim, S. K. Hahn, K. Choi, I. C. Kwon, S. Y. Jeong, K. Kim, Angewandte Chemie 2012, 51, 11836-11840; e) R. Rossin, P. R. Verkerk, S. M. van den Bosch, R. C. Vulders, I. Verel, J. Lub, M. S. Robillard, Angewandte Chemie 2010, 49, 3375-3378.

[11] a) G. Bratthall, P. Lindberg, A. Havemose-Poulsen, P. Holmstrup, L. Bay, G. Söderholm, O. Norderyd, B. Andersson, B. Rickardsson, H. Hallström, B. Kullendorff, H. Sköld Bell, Journal of clinical periodontology 2001, 28, 923929; b) A. Bent, R. Tutrone, M. McLennan, L. Lloyd, M. Kennelly, G. Badlani, Neurourology and urodynamics 2001, 20,157-165; c) G. Bratthall, P. Lindberg, A. Havemose-Poulsen, P. Holmstrup, L. Bay, G. Soderholm, O. Norderyd, B. Andersson, B. Rickardsson, H. Hallstrom, B. Kullendorff, H. Skold Bell, Journal of clinical periodontology 2001, 28, 923-929; d) C. Dawson, M. W. Armstrong,

S. C. Fulford, R. M. Faruqi, R. B. Galland, Journal of the Royal College of Surgeons of Edinburgh 1992, 37, 177-179; e) R. B. Elliott, L. Escobar, P. L. Tan, M. Muzina, S. Zwain, C. Buchanan, Xenotransplantation 2007, 14, 157$161 ;$ f) L. C. Lee, Z. Zhihong, A. Hinson, J. M. Guccione, Journal of visualized experiments : JoVE 2013; g) T. A. Selmi, P. Verdonk, P. Chambat, F. Dubrana, J. F. Potel, L. Barnouin, P. Neyret, The Journal of bone and joint surgery. British volume 2008, 90, 597-604. 
[12] a) R. R. Chen, E. A. Silva, W. W. Yuen, D. J. Mooney, Pharmaceutical research 2006, 24, 258-264; b) E. A. Silva, D. J. Mooney, Biomaterials 2010, 31, 1235-1241.

[13] F. M. Balis, J. S. Holcenberg, W. A. Bleyer, Clinical pharmacokinetics

1983, 8, 202-232.

[14] M. R. Karver, R. Weissleder, S. A. Hilderbrand, Bioconjugate chemistry

2011, 22, 2263-2270

[15] a) C. A. Cezar, S. M. Kennedy, M. Mehta, J. C. Weaver, L. Gu, H.

Vandenburgh, D. J. Mooney, Advanced healthcare materials 2014; b) X. Zhao,

J. Kim, C. A. Cezar, N. Huebsch, K. Lee, K. Bouhadir, D. J. Mooney,

Proceedings of the National Academy of Sciences of the United States of

America 2011, 108, 67-72.

[16] S. Mura, J. Nicolas, P. Couvreur, Nature materials 2013, 12, 991-1003. 


\section{Entry for the Table of Contents}

Selective targeting of small molecules to intramuscular and intradermal sites is achieved through click chemistry. Click-mediated targeting exhibited a high degree of specificity for the target sites and the targeting could be repeated over at least one month through nine administrations. Spatial resolution of two different molecules can be achieved by targeting them to two different sites in the same animal by use of orthogonal click chemistries,establishing the possibility of combining incompatible therapies in patients.

\section{COMMUNICATION}

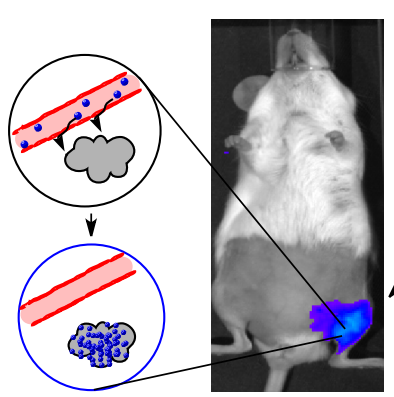

$\mathrm{R}_{1} \gg$

$\pi \mathrm{NH}$

IV small

molecules

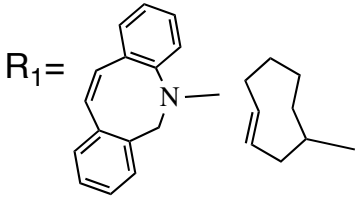

$\underbrace{R \text { gel }}_{\text {implanted }}$
Yevgeny Brudno, Brian J Kwee, Rajiv R Desai, Neel S Joshi, Michael Aizenberg, and David J Mooney*

Page No. - Page No.

In Vivo Targeting through Click Chemistry 